\title{
Perfil dos pacientes e características do tratamento fisioterapêutico aplicado aos trabalhadores com LER/DORT em Juiz de Fora, MG
}

\author{
Profile of patients and physiotherapeutic treatment characteristics applied \\ to workers with RSI/WMSDS in the city of Juiz de Fora, MG
}

\author{
Vanusa Caiafa Caetano ${ }^{[a]}$, Danielle Teles da Cruz ${ }^{[\mathrm{b}]}$, Isabel Cristina Gonçalves Leite ${ }^{[\mathrm{c}]}$
}

[a] Mestre em Saúde Coletiva, Doutoranda em Saúde Brasileira pela Universidade Federal de Juiz de Fora (UFJF), professora assistente II da Faculdade de Fisioterapia da Universidade Federal de Juiz de Fora (UFJF), Juiz de Fora, MG - Brasil, e-mail: vcaiafa@gmail.com

[b] Mestranda em Saúde Coletiva Universidade Federal de Juiz de Fora (UFJF), bolsista FAPEMIG, professora auxiliar I da Faculdade de Fisioterapia da Universidade Federal de Juiz de Fora (UFJF), Juiz de Fora, MG - Brasil, e-mail: danitcruz@yahoo.com.br

[c] Doutora em Saúde Coletiva, professora adjunta do Departamento de Saúde Coletiva da Faculdade de Medicina da Universidade Federal de Juiz de Fora (UFJF), Juiz de Fora, MG - Brasil, e-mail: isabel.leite@ufjf.edu.br

\section{Resumo}

Introdução: A reestruturação do processo produtivo verificada nas últimas décadas implicou alterações diretas na saúde do trabalhador, modificando o perfil de adoecimento e sofrimento desses indivíduos. Dentro desse contexto, observa-se um aumento significativo dos casos de Lesões por Esforços Repetitivos (LER) e dos Distúrbios Osteomusculares Relacionados ao Trabalho (DORT). Objetivo: Conhecer o perfil dos trabalhadores com LER/DORT e a assistência fisioterapêutica aos quais são submetidos. Metodologia: Pela aplicação de um questionário estruturado, foram entrevistados 80 trabalhadores, usuários do SUS, inseridos no Programa de Reabilitação Física do Centro de Referência em Saúde do Trabalhador (Cerest), por meio do Departamento de Saúde do Trabalhador (DSAT), Prefeitura Municipal de Juiz de Fora, Secretaria de Saúde (SS). Resultados: Há um predomínio de casos de DORT no sexo feminino, e em $83,8 \%$ dos casos a renda pessoal não passa de um salário mínimo. Das ocupações encontradas, 76 (95\%) estavam relacionadas com o trabalho braçal, e quanto ao tempo de trabalho em uma mesma função, a média foi de 16 anos. Setenta e oito $(97,5 \%)$ trabalhadores afirmaram impossibilidades na realização das atividades de vida diária (AVDs). Quanto ao tratamento fisioterapêutico, 30 (37,6\%) relataram realizar algum tipo de cinesioterapia. Conclusão: A visão estritamente fisiopatológica, baseada no modelo unidirecional e curativista, não mostram resultados satisfatórios e 
eficazes. Isso implica a não resolução dos quadros álgicos, favorecendo períodos extensos de tratamento fisioterapêutico, sobrecarga do serviço público de saúde e repercussões no âmbito emocional e sócioeconômico desses trabalhadores.

Palavras-chave: Fisioterapia. LER/DORT. Saúde do trabalhador.

\begin{abstract}
Introduction: The restructuring of the production process in recent decades resulted in direct changes in the worker's health, changing the profile of illness and suffering of these individuals. Within this context, there is a significant increase in cases of Repetitive Strain Injury (RSI) and Work-Related Musculoskeletal Disorders (WRMD). Objective: To know the profile of workers with RSI/WRMD and physical therapy to be undergone. Methods: By applying a structured questionnaire, 80 employees were interviewed, users of the SUS (public health system of Brazil), included in the Physical Rehabilitation Program of the Reference Center for Occupational Health (Cerest - Centro de Referência em Saúde do Trabalhador), through the Department of Occupational Health (DSAT - Departamento de Saúde do Trabalhador), City Hall of Juiz de Fora, Department of Health (SS - Secretaria de Saúde). Results: There is a predominance of cases of WRMD in females, and $83.8 \%$ of the cases personal income is only a minimum wage. Within the occupations found, 76 (95\%) were related to manual labor, and the average time working on the same function was of 16 years. Seventy-eight (97.5\%) of workers claimed impossibilities in carrying out activities of daily living (ADL). In the physical therapy, $30(37.6 \%)$ reported that they had some kind of kinesiotherapy. Conclusion: The strictly pathophysiological vision based on the unidirectional and curative model, does not shown satisfactory and effective results. This fact implies no resolution in pain conditions, favoring extended periods of physical therapy, the overload of public system of health and impact on the emotional and socio-economic development of these workers.
\end{abstract}

Keywords: Physical therapy. RSI-WRMD. Worker's health.

\title{
Introdução
}

A adoção de novas tecnologias e métodos gerenciais, verificada nas últimas décadas, facilitou a intensificação do trabalho que, aliada à instabilidade no emprego, modificou o perfil de adoecimento e sofrimento dos trabalhadores. Esse perfil é expresso, entre outros fatores, pelo aumento da prevalência de doenças relacionadas ao trabalho, o surgimento de novas formas de adoecimento mal caracterizadas, como o estresse e a fadiga física e mental, dentre outras manifestações de sofrimento relacionadas ao trabalho (1).

No Brasil, a síndrome de origem ocupacional foi reconhecida pelo Ministério da Previdência e Assistência Social como Lesões por Esforços Repetitivos (LER), por meio da Norma Técnica de Avaliação de Incapacidade. Em 1997, com a revisão desta norma foi introduzida a expressão Distúrbios Osteomusculares Relacionados ao Trabalho (DORT) (2).

A instrução normativa do Instituto Nacional de Seguridade Social (INSS) usa a expressão LER/ DORT, estabelecendo um conceito da síndrome, declarando que ela não se origina exclusivamente de movimentos repetitivos, podendo ocorrer pela permanência prolongada dos segmentos corporais em determinadas posições, assim como a necessidade de concentração e atenção do trabalhador para realização das atividades laborais e a pressão imposta pela organização do trabalho (3).

As LER/DORT representam o principal grupo de agravos à saúde entre as doenças ocupacionais no País (4). Podem ser definidas como manifestações ou síndromes patológicas que se instalam insidiosamente em determinados segmentos do corpo em consequência do trabalho realizado de forma inadequada, de acordo com as exigências das tarefas, ambientes físicos e com o processo da organização do trabalho (5). São consideradas como expressões de desequilíbrio entre as exigências do trabalho e as possibilidades humanas, 
tanto no que se refere à capacidade física como mental. O resultado dessa desproporcionalidade é um grande contingente de trabalhadores jovens, com dores crônicas, incapacitados, na maioria das vezes, no auge da sua força de trabalho, colocados à margem de qualquer possibilidade de ascensão social (3).

De acordo com os apontamentos de Garbin, Neves e Batista (6), os trabalhadores com LER/DORT se deparam com várias dificuldades diariamente, tais como dor, limitações físicas, desrespeito, desconfiança por parte dos empregadores, humilhação e menosprezo, observados na relação com médicos peritos do INSS, incompreensão da família, perda da capacidade laboral, e outras situações.

Sendo assim, éimprescindível a compreensão do significado global deste processo saúde-adoecimento, considerando as condições sociais e culturais desses trabalhadores, uma vez que a presença da doença desencadeia veementemente a interferência dos padrões de visibilidade complexa e contínua da sociedade, seja no individual ou no coletivo (7). Partindo dessas premissas e das demandas instaladas atualmente, o fisioterapeuta configura-se como importante colaborador da Justiça do Trabalho $(8,9)$.

Amparado na Resolução n. 259, de 18 de dezembro de 2003, do Conselho Federal de Fisioterapia e Terapia Ocupacional (COFITTO), cabe ao fisioterapeuta do trabalho identificar, avaliar e observar os fatores ambientais que possam constituir risco à saúde funcional do trabalhador, em qualquer fase do processo produtivo, alertando a empresa sobre sua existência e possíveis consequências; realizar a análise biomecânica da atividade produtiva do trabalhador, considerando as diferentes exigências das tarefas nos seus esforços estáticos e dinâmicos; entre outras atribuições. Em 13 de junho de 2008, a Resolução n. 351 do COFFITO reconheceu a Fisioterapia do Trabalho como especialidade do profissional fisioterapeuta (9).

A Saúde do Trabalhador é um campo específico da área da Saúde Pública que procura atuar por meio de procedimentos próprios, com a finalidade de promover e proteger a saúde de pessoas envolvidas no exercício do trabalho. Ela se constrói por meio do desenvolvimento de ações de vigilância dos riscos presentes nos ambientes e condições de trabalho, dos agravos à saúde do trabalhador e de organização e prestação da assistência aos trabalhadores, compreendendo procedimentos de diagnóstico, tratamento e reabilitação de forma integrada, no SUS (10).

Isto implica uma atuação multidisciplinar e interdisciplinar, visando à preservação e à promoção da saúde, com medidas de alcance coletivo (11).

Dentro deste contexto, chama-se a atenção para a Fisioterapia, enquanto ramo da saúde que estuda, avalia, previne e trata os distúrbios da cinesia humana decorrentes de alterações de órgãos e sistemas, responsável pela reintegração do paciente à sociedade (12).

Este estudo teve como objetivo conhecer o perfil dos trabalhadores com LER/DORT e a assistência fisioterapêutica aos quais são submetidos no âmbito do serviço público de saúde do município de Juiz de Fora, polo macrorregional de assistência da zona da mata mineira.

\section{Materiais e métodos}

O presente estudo foi realizado com uma amostra de trabalhadores afastados, usuários do SUS, inseridos no Programa de Reabilitação Física do Centro de Referência em Saúde do Trabalhador (Cerest), por meio do Departamento de Saúde do Trabalhador (DSAT), Prefeitura Municipal de Juiz de Fora, Secretaria de Saúde (SS) do município de Juiz de Fora, MG. Este serviço tem como característica atender os trabalhadores com doenças relacionadas ao trabalho ou que sofreram algum tipo de acidente de trabalho, e desenvolver um programa especial de atendimento e assistência específica para eles.

De acordo com os registros consultados do Cerest, encontramos um número de 1.800 trabalhadores cadastrados, entre acidentes de trabalho e doenças relacionadas ao trabalho. A maioria deles se encontra em afastamento ou desempregados, tendo sua doença comunicada ao INSS pela Comunicação de Acidente de Trabalho (CAT).

Os trabalhadores são encaminhados ao Cerest por profissionais de saúde (público e privado), sindicatos, Ministério do Trabalho, Justiça do Trabalho, Instituto Nacional do Seguro Social (INSS), Unidades Básicas de Saúde (UBS), empresas, outras instituições e demanda espontânea. 
Para inserção no Programa de Reabilitação Física do Cerest, esses trabalhadores devem ter uma renda mensal de até três salários mínimos, não havendo a obrigatoriedade de se ter algum vínculo empregatício, podendo ser autônomo, formal ou informal. Ao serem inseridos, os trabalhadores recebem orientações de assistentes sociais, consultas médicas, tratamentos psicológicos e fisioterapêuticos. Ao serem encaminhados para a assistência fisioterapêutica, os trabalhadores recebem mensalmente vales-transporte, dentro de uma trajetória de intervenções realizadas pelo Cerest, geralmente marcadas pela falta de atendimento adequado, seja na rede pública ou na rede privada.

Nesse contexto, como critérios de inclusão para o estudo, selecionamos sujeitos de ambos os sexos, com idade entre 20 e 55 anos, com nexo ocupacional de LER/DORT, cadastrados e frequentes no Programa de Reabilitação Física do Cerest desde o ano de 2006, que se encontravam em tratamento fisioterapêutico e ainda frequentes no momento da pesquisa.

Os critérios de exclusão utilizados foram: trabalhadores com incapacidade de compreensão ou resposta para o questionário utilizado, com doenças associadas (neurológicas e mentais) e os afastamentos caracterizados como acidentes de trabalho.

Com relação à história do trabalho atual, as ocupações foram catalogadas de acordo com os 21 itens de ocupação principal utilizados pela Receita Federal apud Furia (13), e que posteriormente foram reagrupadas.

Nessa perspectiva, o conhecimento do senso comum e a inserção social nas relações que vão definir a assistência fisioterapêutica entre os trabalhadores com LER/DORT pontuam-se a partir da interação dos diferentes trabalhadores envolvidos, em situações concretas.

Optamos por uma abordagem metodológica transversal, quantitativa e descritiva, que teve como ótica atender aos objetivos propostos no presente estudo. Foi aplicado de forma individual, a partir da adesão espontânea dos trabalhadores selecionados, no Cerest, um questionário estruturado, com questões fechadas, desenvolvido e baseado no Roteiro de Investigação para Abordagem Ergonômica das Doenças MúsculoEsqueléticas, do Ministério da Saúde (2). Esse instrumento ainda compôs os respectivos blocos de: identificação, história social, história do trabalho atual, história da doença atual, avaliação clínica (história clínica, história familiar), questões relacionadas à assistência fisioterapêutica e LER/DORT, no intuito de identificar e conhecer o perfil dos trabalhadores estudados.

Os dados foram analisados produzindo medidas descritivas com o apoio do software Statistical Package for the Social Sciences $\left(\right.$ SPSS $\left.^{\circledR}\right)$ versão 8.0.

Toda a metodologia foi desenvolvida obedecendo aos princípios éticos da Resolução 196/96 do Conselho Nacional de Saúde. O presente estudo foi analisado e aprovado pelo Comitê de Ética em Pesquisa (CEP) da Universidade Federal de Juiz de Fora, sob o Parecer n. 326/2007, Protocolo n. $1213.259 / 2007$.

\section{Resultados e discussão}

No Programa de Reabilitação Física do Cerest há uma frequência média de 300 trabalhadores por mês, caracterizados como vítimas de acidentes de trabalho e doenças ocupacionais. Participaram do nosso estudo 80 trabalhadores, e dez recusaram a participação, conforme Figura 1.

$\mathrm{Na}$ presente amostra, 93,8\% dos trabalhores são do sexo feminino. Galete (14) acredita que tal achado deve-se ao desemprego dos homens, que leva as mulheres a procurarem opções diferenciadas para complementar a renda familiar. Com relação à escolaridade, $32(40,1 \%)$ trabalhadores tinham ensino fundamental de $1^{\mathrm{a}}$ à $4^{\mathrm{a}}$ série e, respectivamente, o mesmo percentual para a $5^{\mathrm{a}}$ à $8^{\mathrm{a}}$ série; $15(18,8 \%)$ ensino médio e um $(1,3 \%)$ trabalhador com ensino superior incompleto. Esses dados corroboram com os achados na literatura (15).

De acordo com a história social, com relação ao estado civil dos trabalhadores, 48 (60\%) eram casados, $8(10 \%)$ solteiros, $14(17,5 \%)$ separados e 10 (12,5\%) encontravam-se em outras situações (união consensual). O número de moradores por residência foi até quatro pessoas em 76,4\% (61) e 23,8\% (19) acima de quatro moradores. A Tabela 1 demonstra os dados socioeconômicos dos trabalhadores. 


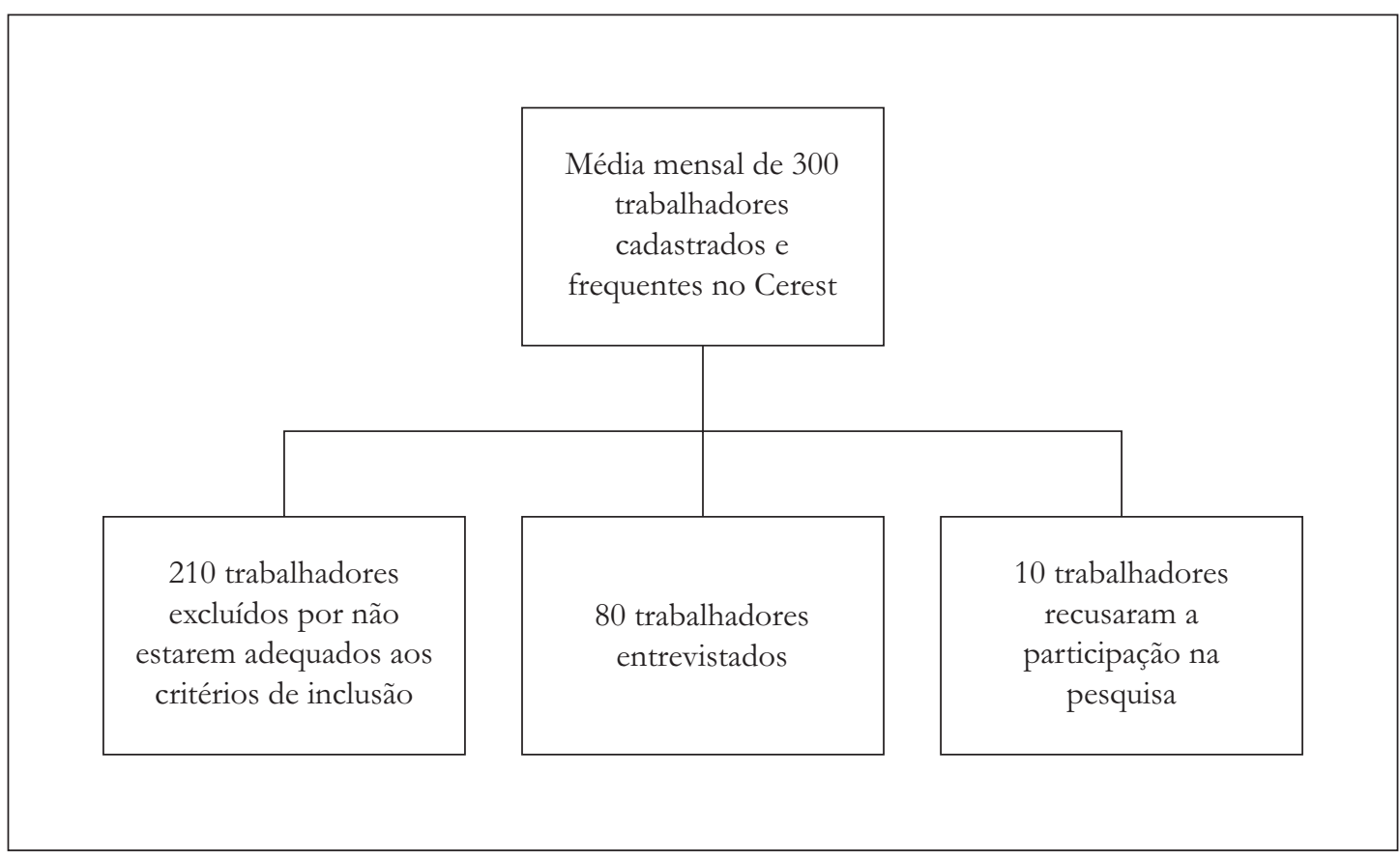

Figura 1 - Organograma representativo da seleção dos sujeitos da pesquisa

Tabela 1 - Dados sócio-econômicos dos trabalhadores do CEREST de Juiz de Fora, MG, 2007-2008

\begin{tabular}{lccc}
\hline Dados demográficos & Categorias & Frequência & Percentual (\%) \\
\hline Condições de moradia & Própria & 48 & 60 \\
& Alugada & 17 & 21,3 \\
& Cedida & 14 & 17,5 \\
Renda pessoal & Até 1 S.M.* & 67 & 83,8 \\
& Acima de 2 S.M. & 2 & 2,6 \\
& Sem renda & 11 & 13,8 \\
Renda familiar & 1 a 3 S.M. & 67 & 83,8 \\
& 3 a 5 S.M. & 12 & 15 \\
& Não sabe & 1 & 1,3 \\
\hline
\end{tabular}

*S.M. = Salário Mínimo.

Das ocupações encontradas, 76 (95\%) estavam relacionadas com o trabalho braçal, prevalecendo neste reagrupamento os trabalhadores das indústrias têxteis, do curtimento, do vestuário, seguidos dos trabalhadores técnico-administrativos, com três (3,8\%), e do comércio, com um (1,3\%). Quanto ao vínculo empregatício, $71(88,8 \%)$ eram trabalhadores formais, seis $(7,5 \%)$ autônomos e três $(3,8)$ terceirizados. Com 
relação ao tempo de função em que esses trabalhadores se encontravam no momento da pesquisa, os dados mostram 16 anos de trabalho, em média, na mesma função. De acordo com os apontamentos da literatura, a organização do processo de trabalho e a redistribuição das atividades laborais são elementos centrais para a garantia da saúde e qualidade vida do trabalhador. Dentro desse campo, a ergonomia é um dos componentes que permitem a reorganização e a diversificação das atividades laborais, respeitando os limites e peculiaridades de cada trabalhador e função. Essa concepção tende a valorizar a especificidade do funcionamento humano, não subestimando a influência da rigidez nos processos de organização do trabalho (16).

No que se refere à situação profissional, todos os trabalhadores encontravam-se em afastamento ocupacional, com uma média de quatro anos de afastamento, mínimo de dois anos e máximo de oito anos. A perda salarial foi relatada por $55(68,8 \%)$ trabalhadores, em função do afastamento laboral.

Dentre os acidentes de trabalho mencionados, 18 (22,5\%) já sofreram algum tipo de acidente, como cortes, ferimentos, queimaduras, traumas e fraturas. Estes não foram relacionados com o afastamento ocupacional atual.

Quanto à carga horária de trabalho, a média diária foi de 9 horas, com máxima de 15 horas/dia. A média de idade dos trabalhadores foi de 45 anos, e a média de idade que os mesmos iniciaram suas vidas profissionais foi de 12 anos, sendo a mínima descrita em seis anos de idade, ambos considerados como trabalho infantil. Pouca atenção tem sido dada aos adultos que foram expostos a certas condições de trabalho quando crianças. Nesse sentido, não se conhece muito sobre a cascata de eventos que podem dar resultados adversos no sistema músculo-esquelético (17).

Além de exercerem suas atividades laborais, 54 (67,5\%) trabalhadores realizavam outros tipos de serviços ("bicos" e "serviços do lar") antes do afastamento ocupacional, configurando uma dupla jornada de trabalho.

Em relação ao tempo de dor relatado pelos trabalhadores, os dados mostram uma média de seis anos, chegando a 26 anos em seu valor máximo. A caracterização das LER/DORT demorou em média dois anos entre o início dos sintomas e o estabelecimento no nexo causal. O tempo mínimo para esta conclusão foi de um ano e o máximo de 13 anos. A dor é um sintoma comum na população e de alta prevalência, considerado um problema de saúde pública mundial (18). É capaz de gerar sofrimento, incapacidade, diminuição da capacidade laboral e, consequentemente, impacto significativo na produtividade econômica $(18,19)$.

Segundo os diagnósticos médicos ocupacionais apresentados pelos trabalhadores pesquisados, verificamos uma maior prevalência nas combinações de LER/DORT (57,5\%), que se referem a uma combinação de vários diagnósticos, tais como: tendinites, tenossinovites, lombalgias, cervicalgias, hérnias de disco, Síndrome do Túnel do Carpo, Tenossinovite estenosante de Quervain, conforme a Tabela 2.

É importante mencionar que os médicos diagnosticam segundo as afecções previstas pela Previdência Social Brasileira, a qual não reconhece a fibromialgia, a Síndrome Fascial, mas prevê a cervicobraquialgia e a cervicalgia (20). Os quadros clínicos encontrados no presente estudo foram localizados em membros superiores, o que corrobora com os relatados na literatura (21), exceto três trabalhadores que apresentaram lombalgia associada à hérnia de disco.

Tabela 2 - Diagnósticos ocupacionais dos trabalhadores investigados

\begin{tabular}{lcc}
\hline Diagnóstico Ocupacional & Frequência & Porcentual (\%) \\
\hline Tendinite & 30 & 37,5 \\
Síndrome do Túnel do Carpo & 1 & 1,3 \\
Lombalgia (hérnia de disco) & 3 & 3,8 \\
Combinações de DORT & 46 & 57,5 \\
\hline
\end{tabular}


Quando questionados quanto às limitações das atividades da vida diária (AVDs), 78 (97,5\%) trabalhadores afirmaram impossibilidades de realizá-las, e 44 (55\%) trabalhadores já haviam sido orientados sobre a melhor forma de executá-las, sendo $27(33,8 \%)$ por fisioterapeutas, $17(21,3 \%)$ por médicos e dois $(2,5 \%)$ por outros meios. Augusto et al. (22) destacaram a importância da prática fisioterapeutica quanto às orientações relacionadas aos cuidados posturais, bem como posicionamento e cuidados nas AVDs, sendo também imprescindível para melhor qualidade do tratamento e prevenção das LER/DORT. Doravante, não foi isso verificado no presente estudo, o que reflete a inadequação de práticas de prevenção, promoção e educação em saúde.

Tais habilidades têm sido alvo de preocupação internacional, com crescente interesse entre os profissionais de saúde, pelo fato de que a incapacidade funcional para as AVDs é preditora de demência, fragilidade e até mesmo mortalidade (23). Segundo referencial teórico de Assunção (24), tais limitações podem ser classificadas como estágio máximo das LER/DORT, conhecido como fase 4, que se caracterizam pela impossibilidade de realização das tarefas domésticas e trabalho, dificuldade de dormir ocasionada pela dor, limitação dos movimentos, atrofia e/ou deformidades.

$\mathrm{Na}$ avaliação clínica, $44(55 \%)$ trabalhadores relataram apresentar algum problema de saúde, nos quais episódios combinados (depressão, gastrite, doenças cardiovasculares, diabetes, dentre outros) foram apontados em 19 (23,8\%) trabalhadores, seguido das doenças cardiovasculares em 14 (17,5\%) e outras em oito $(10 \%)$ trabalhadores.

No que diz respeito à assistência fisioterapêutica, os dados mostram que a média com relação ao tempo em que os trabalhadores fazem fisioterapia foi de 4 anos e 6 meses, e que eles frequentaram em média duas clínicas diferentes de fisioterapia. Desses, 59 (73,8\%) trabalhadores recebem assistência fisioterapêutica em locais públicos, seguidos de 19 (23,8\%) representados por múltiplos locais (universitários privados e públi$\cos )$ e dois $(2,5 \%)$ estritamente em locais privados. A frequência semanal das sessões de fisioterapia realizadas de quatro a cinco vezes por semana prevaleceu em $52(65 \%)$ trabalhadores, seguidos de três vezes semanais em 26 (32,5\%). Esses dados corroboram com outros achados deste estudo, no qual 65 (81,3\%) trabalhadores relataram interrupção no tratamento fisioterapêutico mensal, justificada em função do término do número de dez sessões liberadas pelo SUS durante um mês.

Machado et al. (25), em estudo da análise do perfil do setor secundário do serviço de fisioterapia de Juiz de Fora, encontraram dados que corroboram com os nosso achados: dez sessões liberadas pelo SUS mensalmente, tratamento fisioterapêutico com baixa ou ausência de práticas de cinesioterapia, e pouca orientação e supervisão do tratamento por parte dos fisioterapeutas. A alternativa para o enfrentamento de tal problema seria a reorganização do serviço de fisioterapia (incluído numa rede de atenção à saúde), bem como de redirecionamento de investimentos e de adequação aos princípios e diretrizes do SUS.

Para Rodrigues (26), as possibilidades de atuação do fisioterapeuta têm caminhado para maior adequação à política pública de saúde preconizada pelo SUS, na qual a valorização da prevenção e promoção da saúde e maior resolutividade dos atendimentos prestados representam um dos elementos norteadores das ações propostas.

Antes de serem submetidos ao tratamento fisioterapêutico, 58 (72,5\%) trabalhadores relataram que são avaliados por fisioterapeutas antes do início das sessões. Todos os trabalhadores souberam informar quais os procedimentos fisioterapêuticos são utilizados nas sessões de fisioterapia, nas quais 30 (37,6\%) realizavam algum tipo de cinesioterapia. Destes, 13 (16,3\%) realizavam com auxílio do fisioterapeuta, seguidos de 17 $(21,3 \%)$ sem essa ajuda. Na Tabela 3 apresentamos os procedimentos utilizados na assistência fisioterapêutica, segundo relato desses trabalhadores com LER/DORT.

Ao serem questionados quanto ao desejo de interromperem o tratamento fisioterapêutico, caso não fosse uma recomendação da perícia médica, $16(20 \%)$ trabalhadores afirmaram que gostariam, justificando não alcançar melhora nos quadros álgicos, por não gostarem e acharem cansativo. Em contrapartida, $64(80 \%)$ trabalhadores afirmaram que não gostariam de interromper a assistência prestada pela fisioterapia, por sentirem alívio $(39 ; 48,8 \%)$, melhora $(26 ; 32,5 \%)$ e por considerarem-na necessária $(3 ; 3,8 \%)$. 
Tabela 3 - Procedimentos utilizados na assistência fisioterapêutica

\begin{tabular}{lcc}
\hline Procedimento Fisioterapêutico & Frequência & Percentual \% \\
\hline Termoterapia + eletroterapia & 41 & 51,3 \\
Hidroterapia & 3 & 3,8 \\
Hidroterapia + recursos físicos & 6 & 7,5 \\
Cinesioterapia + recursos físicos & 29 & 36,3 \\
Hidroterapia + cinesioterapia + recursos físicos & 1 & 1,3 \\
\hline
\end{tabular}

A recomendação da cinesioterapia é um aspecto importante para a saúde e qualidade de vida do trabalhador (27). No entanto, os resultados obtidos no presente estudo mostram que os tratamentos fisioterapêuticos pouco se baseiam nessa abordagem, desconsiderando a necessidade de uma intervenção eficaz, o que poderá gerar a persistência das dores músculo-esqueléticas e o insucesso na reabilitação dos trabalhadores com LER/ DORT. De acordo com dados da literatura científica, sabemos que são possíveis inúmeras possibilidades de tratamentos fisioterapêuticos que permitem resultados positivos, como: redução do quadro álgico, melhora do estado geral e funções físicas, diminuição do absenteísmo, ganho de força muscular e resistência, melhora no controle motor, desempenho físico, diminuição do estresse ocupacional e aumento da produção $(22,27,28)$.

No entanto, acreditamos também que uma prerrogativa importante para a não resolução do processo de reabilitação dos trabalhadores reside na dificuldade de liberação dos tensionamentos excessivos obtidos nas atividades laborais, o que favorece a não reabilitação de estruturas lesionadas (22).

Há um consenso entre os fisioterapeutas de que a meta principal de qualquer intervenção é a melhoria da condição funcional e, consequentemente, da qualidade de vida dos indivíduos. Os interesses das pesquisas recentes vão além dos relacionados a componentes físicos como amplitude de movimento, força muscular e capacidade aeróbica, e dão espaço a investigações referentes à qualidade de vida e aos níveis de função dos indivíduos (29).

Cabe ressaltar ainda, segundo Ikari (28), que a atuação de uma equipe multidisciplinar é essencial para obtenção de resultados positivos. Sendo assim, entendendo a complexidade do processo de saúde-doença, devemos buscar o trabalho multidisciplinar, a autonomia de todos os profissionais de saúde e troca constante de conhecimentos e saberes (28).

\section{Considerações finais}

De acordo com o presente estudo, é notório que há um predomínio de casos de LER/DORT no sexo feminino e em classes econômicas menos favorecidas, corroborando com os achados na literatura $(21,30)$, o que nos chama atenção para uma abordagem ampliada e contextualizada desses trabalhadores. A visão estritamente fisiopatológica, baseada no modelo unidirecional e curativista, não mostram resultados satisfatórios e eficazes - fato esse que implica a não resolução dos quadros álgicos, favorecendo períodos extensos de tratamento fisioterapêutico, sobrecarga dos serviços públicos de saúde e repercussões no âmbito emocional e socioeconômico desses trabalhadores

Outro fato que chama atenção é com relação à inadequação do tratamento fisioterapêutico utilizado para esses pacientes e à falta de uma abordagem interdisciplinar, que repercute em não resolução dos quadros álgicos, períodos extensos de tratamento fisioterapêutico, sobrecarga do serviço público de saúde e repercussões no âmbito emocional e socioeconômico desses trabalhadores. 
Adicionalmente torna-se necessária a ampliação na formação de fisioterapeutas do conhecimento e da percepção dos impactos e dos efeitos adversos de determinados ambientes de trabalho sobre o aparato musculoesquelético. Uma vez que esses profissionais têm se deparado frequentemente com trabalhadores que apresentam lesões com envolvimento ocupacional.

Ainda resta muito a ser estudado e pesquisado acerca desses trabalhadores adoecidos. Podemos afirmar que esses acometimentos já são considerados epidêmicos, de extrema relevância de saúde pública, e exigem a atenção de profissionais de vários campos de trabalho. Reforçamos a importância de pesquisas com enfoque interdisciplinar que possibilitem encontrar meios para auxiliar esses trabalhadores, com intuito de aproximar as diversas áreas de atuação, bem como estudos voltados para o tratamento fisioterapeutico e que possam avaliar as formas e meios de atuação mais eficazes.

\section{Referências}

1. Brasil. Ministério da Previdência e Assistência Social. Lista de Doenças Relacionadas ao Trabalho. Brasília: MTE/ SIT; MPAS; 2001.

2. Brasil. Ministério da Saúde. Representação no Brasil da OPAS/OMS. Doenças relacionadas ao trabalho: manual de procedimentos para os serviços de saúde. Brasília: Ministério da Saúde; 2001.

3. Brasil. Ministério da Saúde. Secretaria de Atenção à Saúde. Departamento de ações programáticas estratégicas. Lesões por esforços repetitivos (LER) distúrbios osteomusculares relacionados ao trabalho (DORT). Ministério da Saúde, Secretaria de Atenção à Saúde, Departamento de Ações Programáticas Estratégicas. Brasília: Ministério da Saúde; 2005.

4. Ragasson CAP. Qualidade no trabalho: estudo das condições de trabalho. Cascavel: Coluna do Saber; 2004.

5. Pivetta AD, Jacques MA, Agne JE, Lopes LF. Prevalência de distúrbios osteomusculares relacionados ao trabalho em fisioterapeutas. Revista Digital - Buenos Aires. 2005;10(80).

6. Garbin AC, Neves IR, Batista RM. Etiologia do senso comum: as Lesões por Esforços Repetitivos na visão dos portadores. Cad Psico Soc Trab. 1998;1(1):43-5.

7. Arouca S. O dilema preventivista: contribuição para a compreensão e crítica da medicina preventiva. São Paulo: UNESP; Rio de Janeiro: FIOCRUZ; 2003.

8. Brasil. Ministério do Trabalho e Emprego. Classificação brasileira de ocupações, códigos, títulos e descrições. 3. ed. Brasilia: Ministério do Trabalho e Emprego; 2010.

9. Baú LM, Klein AA. O reconhecimento da especialidade em fisioterapia do trabalho pelo COFFITO e Ministério do Trabalho/CBO: uma conquista para a fisioterapia e a saúde do trabalhador. Rev Bras Fisioter. 2009;13(2):5-6.

10. Brasil. Ministério da Saúde do Brasil. Representação no Brasil das doenças relacionadas ao trabalho: manual de procedimentos OPAS/OMS para os serviços de saúde. Brasília: Ministério da Saúde; 2001.

11. Minayo GC, Thedim CSM. A construção do campo de saúde do trabalhador: percursos e dilemas. Cad Saúde Pública. 1997;13(2):21-32.

12. Rebelatto JR, Botomé SP. Fisioterapia no Brasil: fundamentos para uma ação preventiva e perspectivas profissionais. 2a ed. São Paulo: Manole; 1999.

13. Furia CLB. Qualidade de vida em pacientes tratados de câncer de cavidade oral, faringe e laringe em São Paulo: estudo multicêntrico [tese]. São Paulo: Universidade de São Paulo; 2006.

14. Galete RA. Mercado de trabalho formal dos serviços pessoais e domiciliares no Brasil. Rev Perspec Contemp. 2006;1(1):1-31. 
15. Costa EDGMM, Arias AJ, Oliveira SM, Nichols OC. Prevalência de síndromes dolorosas osteomusculares em trabalhadores de Unidades Básicas de Saúde (UBS). Einstein. 2007;5(1):37-43.

16. Guérin F, Laville A, Daniellou F, DuraffourgJ, Kerguelen A. Compreender o trabalho para transformá-lo. A prática da ergonomia. São Paulo: Edgard Blücher; 2001.

17. Selevan SG, Kimmel CA, Mendola P. Identifying critical windows of exposure for children health. Environ Health Perspect. 2000;108(Suppl 3):451-55.

18. Blyth FM, March LM, Brnabic AJM, Jorm LR, Williamson M, Cousins MJ. Chronic pain in Australia: a prevalence study. Pain. 2001;89(2/3):127-34.

19. Coury HJCG, Walsh IA, Alem M, Oishi J. Influence of gender on work-related musculoskeletal disorders in repetitive tasks. Int J Ind Erg. 2002;29(1):33-9.

20. Assunção A, Almeida IM. Doenças osteomusculares relacionadas com o trabalho: membro superior e pescoço. In: Mendes R. (Org.). Patologia do trabalho: atualizada e ampliada. 2a ed. Rio de Janeiro: Atheneu; 2003.

21. Ghisleni AP, Merlo ARC. Trabalhador contemporâneo e patologias por hipersolicitação. Psicol Reflex Crít. 2005;18(2):171-76.

22. Augusto VG, Sampaio RF, Tirado MGA, Mancini MC, Parreira VF. Um olhar sobre as LER/DORT no contexto clínico do fisioterapeuta. Rev Bras Fisioter. 2008;12(1):49-56.

23. Raso V. A adiposidade corporal e a idade prejudicam a capacidade funcional para realizar as atividades da vida diária de mulheres acima de 47 anos. Rev Bras Med Esporte. 2002;8(6):225-34.

24. Assunção AA. Sistema músculo-esquelético: Lesões por Esforços Repetitivos (LER). In: Mendes R. Patologia do trabalho. Rio de Janeiro: Atheneu; 1995. p. 173-98.

25. Machado GLR, Fayer VA, Caldas MAJ, Cruz DT. Análise do perfil do serviço de fisioterapia do setor secundário do SUS de Juiz de Fora, MG. Anais do IX Congresso Brasileiro de Saúde Coletiva; 2009; Recife, Brasil. Juiz de Fora, UFJF; 2008.

26. Rodrigues RM. A fisioterapia no contexto da política de saúde no Brasil: aproximações e desafios. Perspectivas. 2008;2(8):104-9.

27. Assunção AA, Vilela LVO. Lesões por esforços repetitivos: guia para profissionais de saúde. Piracicaba: CEREST; 2009.

28. Ikari TE, Mantelli M, Corrêa Filho HR, Monteiro MI. Tratamento de LER/DORT: intervenções fisioterápicas. Rev Ciênc Med. 2007;16(4-6):233-43.

29. Mendonça FM, Trindade FMG, Oliveira L, Sampaio RF, Silva FCM, Alves GBO. Ginástica e sintomas ostemomusculares em trabalhadores de uma indústria têxtil de Minas Gerais. Fisioterapia Brasil. 2004;5(6):425-30.

30. Merlo ARC, Jacques MGC, Hoefel MGL. Trabalho de grupo com portadores de LER/DORT: um relato de experiência. Psicol Reflex Crít. 2001;14(1):253-58.

Recebido: 19/03/2009

Received: 03/19/2009

Aprovado: 25/01/2010

Approved: 01/25/2010

Revisado: 24/06/2010

Reviewed: 06/24/2010 